\title{
Voxel Size
}

National Cancer Institute

\section{Source}

National Cancer Institute. Voxel Size. NCI Thesaurus. Code C95003.

The three distances on the imaged subject that correspond to the width, height, and depth of the smallest piece of the subject that is displayed as a voxel. 